\title{
Regards sociologiques sur la dynamique du lien social dans les sociétés de joutes languedociennes
}

Charles Pigeassou et Jérôme Pruneau

\section{(2) OpenEdition \\ 1 Journals}

Édition électronique

URL : http://journals.openedition.org/corpsetculture/509

ISSN : $1777-5337$

Éditeur

Association Corps et Culture

Édition imprimée

Date de publication : 1 juin 1998

ISSN : 1268-5631

\section{Référence électronique}

Charles Pigeassou et Jérôme Pruneau, «Regards sociologiques sur la dynamique du lien social dans les sociétés de joutes languedociennes », Corps et culture [En ligne], Numéro 3 | 1998, mis en ligne le 12 octobre 2007, consulté le 07 septembre 2020. URL : http://journals.openedition.org/

corpsetculture/509

Ce document a été généré automatiquement le 7 septembre 2020.

(c) tous droits réservés 


\title{
Regards sociologiques sur la dynamique du lien social dans les sociétés de joutes languedociennes
}

\author{
Charles Pigeassou et Jérôme Pruneau
}

1 S'interroger sur la forme et la nature prises par le lien social dans les pratiques de joute, c'est d'une part étudier les processus de « reliance et de déliance » (Bolle de Bal M., 1996) mais c'est, d'autre part, coupler cette perspective avec l'analyse de l'environnement de ces pratiques. La dialectique cohésion/atomisation sociale se nourrit des grandes mutations de la société. Dans cette configuration, l'étude de la dynamique des liens sociaux dans les jeux traditionnels participe d'une approche critique dans la manière d'appréhender et de concevoir le lien social. Le constat contemporain met en évidence que la pratique de la joute, taraudée par l'emprise du paradigme sportif, est entrée dans une ère de fragilisation du lien social.

2 En rebondissant sur différents paradigmes sociologiques formulant une compréhension $\mathrm{du}$ lien social, l'objectif est de montrer comment le substrat étudié est sensible au contexte qui lui permet de s'exprimer. Puisant dans trois siècles d'histoire de la joute languedocienne, les éléments signifiants d'une transformation des conditions d'exercice et des représentations qui sont associées à la joute construisent les jalons d'une archéologie du lien social dans ces pratiques. Pour autant, ce regard sociohistorique sur la constitution du lien social participe d'une démarche épistémologique en mettant en perspective différentes approches. La dynamique du lien social ne se laisse pas capter par une seule approche théorique, au contraire sa complexité ne peut être révélée que par l'éclairage de différents modèles d'analyse de la relation sociale. Plus complémentaires qu'opposées, les quatre approches retenues la théorie de la sociologie classique, la théorie de l'écologie humaine, le structuralisme critique, la sociologie dynamique - investissent le lien social à partir de registres et de concepts spécifiques afin d'établir une trajectoire où se repèrent une histoire et un état du lien social. L'univers des jeux traditionnels a longtemps été considéré comme un lieu d'expression privilégié des relations interindividuelles et constitue un terrain pertinent 
pour l'observation des phénomènes que l'on délimitera, dans le cadre de cet article, à l'étude de la constitution et de la transformation du lien social dans les pratiques de la joute languedocienne.

Le lien social : substrat permettant d'appréhender la morphologie de la structure sociale

Qu'est-ce que le lien social ? Dans une acception générale, le lien social pourrait être défini comme un ensemble de forces (analysant des rapports) ou de caractéristiques (décrivant des traits) ou de mécanismes (étudiant les interactions) qui permettent de relier les individus entre eux et, simultanément, de rattacher chaque individu à une collectivité. Le lien social décrit le mode selon lequel un individu invente en même temps qu'il reproduit son intégration dans les groupes auxquels il participe. Sous cet angle, les théories de la sociologie classique méritent un détour pour appréhender quelle place occupe le lien social dans la compréhension des phénomènes sociaux. Pour Tönnies, Durkheim, Weber ou Simmel - habituellement qualifiés de pères fondateurs la problématique centrale consiste à s'interroger sur le fait que les hommes vivent ensemble et tissent des relations entre eux qui peuvent prendre la forme d'un groupe, d'une communauté ou d'une société. Imprégnés de la pensée positiviste, ces sociologues recherchent les facteurs d'ordre et de cohésion des relations qui s'établissent entre les hommes: l'équilibre social constituant l'hypothèse de base partagée. Cet équilibre dépend de deux ordres de variables. En premier lieu, la cohésion des rapports établis entre les membres d'un groupement humain définit la nature du lien social. En second lieu, la cohérence et la stabilité des représentations collectives circonscrivent les caractéristiques du lien moral. Sur cette base commune, des analyses singulières permettent d'appréhender la construction de la structure sociale: les interactions individuelles dans le groupe (Simmel G., 1979), la relation de l'agent social à l'autorité (Weber M., 1964), le rôle de la conscience collective dans la constitution du lien social (Durkheim E., 1977). Les travaux réalisés par ces sociologues convergent vers un constat qui associe un type de société (traditionnelle ou moderne) à un type de structure sociale (communauté ou société) dans laquelle se différencient les relations sociales. La modernité transformerait la structure sociale traditionnelle en substituant aux principes coopératifs et fusionnels de la communauté ceux d'individualisation et de rationalisation construisant l'état de société. Le passage à la modernité porterait la marque d'un relâchement du lien social et d'une décomposition de l'ordre social. Dans cette perspective, Tönnies (1963) développe l'idée d'une différenciation de l'organisation sociale au cours de son évolution. Il distingue : la communauté et la société. Ce passage du communautaire au sociétaire marque les transformations de l'organisation sociale des sociétés anciennes aux sociétés modernes. Ce qui apparaît caractéristique dans cette distinction, c'est que le sociétaire dispose de moins en moins de repères pour donner un sens unique au lien social. Rapporté à une microsociologie des groupements et des pratiques de jeux traditionnels, quel pouvoir explicatif offrent ces travaux? Les observations menées dans les sociétés de joute (Pigeassou C., 1996) attestent que le lien social traditionnel fondé sur l'appartenance à un groupe historiquement constitué s'essouffle face aux mutations et aux changements de la pratique. Le lien social aurait tendance à se reconstruire et se réinventer selon les circonstances et les environnements. Plus largement dans le sport, le lien social se développerait à partir de la singularité de l'environnement.

Différents travaux de référence décrivent comment les sociétés ou groupements sportifs agissent sur l'émergence et la construction du lien social. Maurice Agulhon 
(1977) a magistralement montré comment «la vitalité des associations est un bon indice de la sociabilité générale d'une collectivité humaine » dans la France bourgeoise $\mathrm{du}$ xIX ${ }^{\mathrm{e}}$ siècle. Cette analyse de la constitution et du déplacement des formes et des réseaux relationnels a été reprise et adaptée par Pierre Arnaud (1987) pour explorer le développement de la sociabilité sportive. Dans un chapitre du livre Les athlètes de la République, ce dernier caractérise les éléments (conditions objectives, aspects subjectifs) qui expliquent le développement de liens structurés et structurant dans les associations sportives. Il montre comment la vie associative est une réponse à des aspirations d'ordres divers et comment elle les canalise, les façonne et les enrichit. Il distingue une sociabilité spontanée des jeux traditionnels, qui se déroule dans un espace privé agrégeant un nombre limité de personnes entretenant des liens de proximité, d'une sociabilité communautaire qui privilégie les liens relationnels, s'oriente vers l'entre soi et se clôt sur l'intime. La sociabilité sportive, autre forme de sociabilité, se veut publique, dotée de responsables. Elle repose sur une logique distincte dont la pierre angulaire est la scansion du temps: entraînements et compétitions organisent les calendriers. Cette distinction permet à Pierre Arnaud de s'interroger sur l'origine de la sociabilité sportive: est-elle dérivée de la sociabilité traditionnelle par «filiation ou transformations d'autres structures»? Ronald Hubscher (1992) doute de la spécificité de la sociabilité sportive : on ne peut parler de rupture entre les formes d'associations anciennes et nouvelles. Le sport apparaitrait comme une situation prétexte où les activités ne seraient que «l'expression mise au goût du jour d'une sociabilité élitiste ancienne». Cette sociabilité se fonde sur la citoyenneté du participant mais est fondamentalement masculine, marque d'un ostracisme. Jean Camy, étudiant la sociabilité de sociétés givordines (1985), analyse les relations entre les sociétaires lors de différentes activités qui donnent lieu à des relations interpersonnelles. Ces relations instituantes, qui opèrent dans le présent, construisent l'avenir sans pour autant ni renier ni trahir le passé. Ce présent s'installe en établissant le lien entre l'ouverture vers l'extérieur et l'approfondissement de l'intime et de l'entre soi. Pour Jean-Paul Callède $(1985,1987)$, le groupe sportif constitue un important foyer de sociabilité qui se repère par une spécificité et une identité qui s'affichent. Ces éléments traduisent les composantes et les niveaux d'expression de l'intégration $\mathrm{du}$ groupe. Le bénévolat est le mécanisme régulateur de cette socialisation, la référence identitaire fondamentale. Mais, les déterminations extérieures définies par l'appartenance à la communauté sportive offrent au groupe sportif des possibilités de rayonnement. Par delà la vie interne du groupe, l'affiliation au mouvement sportif lui donne l'occasion d'une existence, d'une reconnaissance: la culture sportive se donne à voir, à se distinguer. La sociabilité sportive se développe à partir d'activités formelles - représentées par les réunions, les entraînements, les compétitions et les déplacements - et les activités informelles initiées par les formes de solidarité et de rencontres.

5 Ces travaux historiques et sociologiques insistent sur les différentes facettes des liens sociaux que le concept de sociabilité condense. En exploitant ces perspectives, la modélisation de la sociabilité peut s'établir à partir de deux orientations. La première orientation s'appuie sur des indicateurs précis qui décrivent des caractéristiques des phénomènes évoqués par cette notion: les caractéristiques des réseaux de relations formelles et/ou informelles, le caractère individuel ou collectif, l'intensité ou le mode d'organisation des relations qui peuvent être électives ou affinitaires. La deuxième orientation définit la sociabilité à partir des fonctions repérées dans le système social, 
principalement au nombre de trois: des fonctions captives, des fonctions d'appartenance et des fonctions internes de régulation. Les fonctions captives matérialisent le pouvoir attractif du groupe et fixent des désirs latents ou des demandes explicites de partager des goûts semblables, de participer à la vie sociale. Les fonctions d'appartenance concrétisent le souci aussi bien que l'exigence de représentation et de promotion: il s'agit d'affirmer son identité, son particularisme, son appartenance mais également de constituer un groupe de pression, une force solidaire. Les fonctions internes définissent comment un ensemble de mécanismes prend en charge et régit les rapports interhumains dans le cadre de l'association: assistance, socialisation, civisme, ludisme.... Le lien social développé et tissé par la pratique de la joute et autour de la joute n'apparait qu'une composante d'un lien social plus large. Mais, cette composante constitue un analyseur des effets qu'il induit et qu'il produit.

6 Ce panorama de différentes approches du lien social au niveau macrosociologique et au niveau microsociologique explore la nature du lien social et sa contribution à la compréhension de la structure sociale. Mais, ni l'approche morphologique de la structure sociale, ni l'approche figurative du lien social dans les groupements sportifs n'approfondissent les dimensions dialectiques et dynamiques du lien social, signe de la complexité et de la relativité des phénomènes. Dans ce contexte, la pluralité d'approche et la multiréférentialité semblent opportunes pour comprendre les mécanismes du lien social. En embrassant un point de vue diachronique, une généalogie des formes d'inscription et de régulation des liens sociaux promus par la joute est à construire. De l'indifférenciation à la différenciation, du groupement à l'individu, du défi à la compétition, de la normalisation à la hiérarchie, un ensemble de phénomènes travaillent le lien social à l'œuvre dans cette pratique.

Le lien social dans la pratique de la joute : exploration des formes de regroupement

Selon Durkheim, un groupement d'êtres humains développe des phénomènes d'interactions qui s'étayent en formes spécifiques et solidaires construisant un lien relayé par des représentations collectives associées. L'axe central de cette problématique s'établit électivement sur les influences que la conscience collective introduit dans la nature du lien social. En portant ses analyses sur la division du travail (1978), sur les cadres sociaux intégrateurs (1976) ou la vie religieuse (1979), ce sociologue trace non seulement les formes de regroupements mais il dégage des principes de fonctionnement. Sont attachés à l'œuvre de Durkheim des concepts descriptifs de configuration typique associant lien social et représentation collective (solidarité mécanique, solidarité organique) ou des concepts opératoires pour la compréhension des phénomènes de "reliance » (conscience collective, densité morale, suicide). De façon plus ciblée, les concepts de solidarité mécanique, de solidarité organique et de conscience collective constitueront le cadre référentiel pour observer les phénomènes de « reliance » dans les pratiques de joute.

8 La pratique de la joute a existé dans différentes régions de France où la présence de l'eau a engendré des activités, fixé une population et développé une structure sociale. Elle apparaît au milieu du xiII ${ }^{\mathrm{e}}$ siècle lors de la concentration des troupes du roi SaintLouis dans les ports languedociens pour participer à la huitième croisade : les tournois de joute maintenaient la condition physique des soldats. Des formes de ce jeu perdurèrent et essaimèrent dans différentes régions : Ile de France, région lyonnaise, région provençale, région languedocienne. Historiquement, les pratiques de joute 
resurgissent lors de cérémonies officielles: à Agde en 1601 lors de la visite du gouverneur du Languedoc, à Frontignan en 1629 en l'honneur de la visite du Cardinal Richelieu ou à Sète en 1666 à l'occasion des fêtes de la fondation du port présidées par Colbert. Lors de ces festivités, le mode d'organisation de ces «joustes" révèle des formes de solidarité existantes à un moment donné dans le groupe social. Ainsi, dans une délibération du conseil général d'Agde datée du dernier jour du mois de Mai de l'an 1601, M. de Figaret, premier consul, propose «que le capitaine Antorum Estienne, homme de marine, prenne en charge le vaisseau des mariés et M. Gouson, ou quelque autre jeune homme, celui des jeunes hommes, pour faire les joustes sur la ribière, le plus honorablement et avec autant de lustre que faire se pourra ... » (Pouget V., 1993 : 10). De manière semblable, lors de la fête patronale de la Saint-Louis à Sète, le combat de joutes oppose la troupe de "la Jeunesse " à la troupe des "Mariés", désignée à l'issue de la cérémonie du Pavois ${ }^{1}$. Au cours des quinze jours consacrés aux préparatifs de la fête, un cérémonial est respecté permettant d'accomplir l'ensemble des tâches exigées pour cette manifestation: les bateaux à décorer, les pavois et les lances à préparer, les costumes à mettre en forme, les joueurs d'instrument à trouver, les rameurs à contacter, les jouteurs à initier.... L'organisation de cet événement tisse de nombreuses relations entre les participants, liant les individus pour forger le groupe. Sur le plan sociologique, la constitution des troupes se réalise à partir d'une distinction fondatrice : le statut, jeune ou marié. Ces troupes se décrivent comme des groupements stables et restreints où les hommes sont interchangeables parce qu'ils se ressemblent à la fois du point de vue de leur fonction dans leur groupe respectif et du point de vue de leurs représentations. Ils adhèrent aux mêmes valeurs et partagent les mêmes sentiments. Ainsi, à Sète de 1666 à 1846 lors du Grand prix de la Saint-Louis, les tournois se réitèrent selon le même scénario : la troupe de « la Jeunesse " rassemblant des jeunes gens âgés d'au moins 16 ans est opposée à la troupe des «Mariés ». La victoire collectivement revient à l'une des deux barques. Des prix individuels récompensent les jouteurs qui ont fait tomber à l'eau le plus grand nombre d'adversaires. La pratique s'inscrit dans un ordre social global, elle la renforce plus qu'elle ne la conteste. Bien que cette pratique virile s'incarne dans l'opposition, elle est encadrée par un ensemble de règles où la solidarité domine comme principe de composition et la soumission à l'ordre établi s'érige en loi. Les liens tissés dans ce cadre exprime une forme de solidarité qui relèvent d'une solidarité de type mécanique. Cette configuration dans l'organisation de la pratique décrit une première période où la conscience collective irrigue les consciences individuelles construisant une participation fusionnelle dans le contexte de cette pratique. Les représentations individuelles se construisent à partir d'un projet social promu et encadré par les pouvoirs établis.

9 A partir de 1846 et jusqu'en 1890, la forme organisationnelle de la pratique de la joute se transforme à Sète: les règles constitutives de la formation des groupes qui s'opposent évoluent. La ville s'est développée au cours du XIX siècle à partir de quartiers regroupant des habitants ayant les mêmes origines et travaillant dans les mêmes secteurs d'activités. Principalement, on distingue : le quartier Haut où vivent les familles d'origine italienne tournée vers la pêche en mer des quartiers de la Bordigue et de la Pointe Courte plus cosmopolites dont l'activité est orientée vers la pêche dans l'étang. Cette différenciation dans la ville d'aires culturelles et ethniques est le signe d'une transformation matérielle, elle accompagne un accroissement de la densité de population qui génère une augmentation du flux des relations sociales. Elle marque le 
début de la modernité dans le tissu social. Cet aménagement de la structure sociale se retrouve dans le contexte de la joute: l'organisation des rencontres se bâtit sur une autre modélisation du social. Le Grand prix de la Saint-Louis se dispute le dimanche et oppose des jouteurs «sétois » originaires soit du Quartier Haut soit des quartiers de la Bordigue et de la Pointe Courte. L'identification des participants change de repère : à l'état civil se substitue l'origine et la culture. La différenciation identitaire recoupe à la fois un quartier, une origine ethnique et une profession. La cristallisation du lien social ne tourne plus autour des mêmes polarités. L'ordre générique constitutif des groupes est remplacé par une atomisation naissante. Celle-ci est d'ailleurs renforcée par la méthode de désignation du vainqueur: le tournoi de la Saint-Louis se remporte individuellement. La mutation du collectif à l'individuel renforce le changement. Ces évolutions mettent en évidence la lente ascension de l'individualisme et l'ascendance progressive de la conscience individuelle ou du micro-groupe sur la conscience collective. Le lien social se déplace et reconstruit de nouvelles solidarités qui s'insèrent dans un environnement tribal (le quartier) et, simultanément, adopte un registre plus abstrait de coopération reliant les membres de la société. Dans ce nouveau contexte, le degré de cohérence des représentations collectives s'affaiblit effaçant les repères communautaires au profit de repères sociétaux. Cet état de transformation orienté vers la modernité annonce le passage à une solidarité «organique». Dans la joute, la métaphore organique décrit à la fois l'autonomisation des tournois de quartier qui apparaissent et la participation de tous les quartiers à chacun des tournois.

Une troisième phase s'amorce à partir de 1891, avec la régionalisation des tournois de joute. Que se soit à Agde, Frontignan ou Sète les tournois lors des fêtes votives deviennent régionaux. Cette ouverture spatiale constitue à la fois un élargissement du lien social mais un affaiblissement de celui-ci par une fragmentation. Ainsi, par exemple, l'identification du vainqueur change de registre : l'identification se réalise par la ville au lieu et place du quartier. En passant de l'échelle de la ville à l'échelle de la région, la symbolique de l'espace travaille le lien social. Ce dernier retrouvera, avec l'avènement des sociétés de joute de nouveaux repères de territorialisation.

L'institutionnalisation de la joute : émergence du lien sociétal

11 L'utilisation des travaux de Durkheim sur l'étude du lien social demeure heuristique dans la mesure où l'appareil conceptuel permet de repérer et de comparer des états distincts de la structure sociale globale. Cette perspective transposée à la sphère microsociologique a permis d'appréhender les transformations du lien social à partir du mode d'organisation de la pratique de la joute. La dynamique du lien social observée se coule dans les catégories élaborées par Durkheim sans pour autant se réduire à celles-ci. L'apparition des sociétés de joutes en vagues successives au cours du Xxe siècle est révélatrice de nouvelles condensations du lien social dans cette pratique. La distinction entre deux états devient alors un obstacle à la compréhension de la dynamique. L'approche du lien social selon la théorie de l'écologie humaine semble pertinente pour comprendre à la fois le déplacement et la métamorphose du lien social dans la joute à partir de la métaphore écologique traduisant simultanément des mécanismes de concurrence (compétition) et de communication (coopération). L'Ecole de Chicago (Grafmeyer Y., Joseph I., 1979) a développé une conception de la transformation du lien social en relation avec le développement urbain. La ville est sous l'emprise de processus naturels généraux qui établissent un équilibre au sein des différentes aires naturelles formées par les quartiers. Des forces d'organisation et de désorganisation régulent les groupements d'individus au sein des aires naturelles : ce 
sont particulièrement la mobilité, la densité. Ces notions s'avèrent dignes d'intérêt pour comprendre l'émergence des sociétés de joutes et leur multiplication au cours du $\mathrm{xx}^{\mathrm{e}}$ siècle. Par mobilité, il faut entendre la capacité à se déplacer. Ce mécanisme abolit les distances, facilite la concentration, multiplie les trajets. Il constitue un facteur d'organisation mais il introduit un état d'équilibre instable susceptible d'être réajusté à tout moment. La densité décrit un accroissement de la population entraînant une différenciation et une spécialisation des tâches. Elle apparaît être un facteur d'organisation en fixant et en regroupant sur un même lieu des groupes professionnels, elle devient un facteur de désorganisation dans la mesure où la solidarité traditionnelle fondée sur l'habitude et le sentiment est remplacé par la coopération et l'intérêt.

Mobilité et densité définissent un jeu de forces qui rendent compte de l'évolution contemporaine du lien social dans la joute. Pour la fête locale d'Agde en 1902, " les organisateurs ont reçu environ 80 demandes. Huit villes sont représentées Sète, Frontignan, Mèze, Balaruc, Balaruc-les-Bains, Béziers, Marseillan, Agde. Mais comme 80 jouteurs c'est beaucoup trop, on décide de les tirer au sort pour n'en conserver que 40 » (Pouget V., 1993 : 16). Ce fait montre la dynamique de cette pratique. Le cercle social initial s'élargit en multipliant les contacts mais ce succès s'accompagne d'une densité qui bouleverse les règles habituelles. La pratique de la joute se diffuse dans différentes villes du Languedoc, attirant de plus en plus de jouteurs qui s'inscrivent dans des tournois à partir des annonces lancées et diffusées à l'occasion des fêtes. Cette situation pléthorique se retrouve dans différents tournois organisés au tournant du siècle caractérisant une nouvelle transformation du lien social. Cet état d'instabilité va participer au mouvement de création des sociétés de joute en Languedoc. Trois périodes de création de sociétés peuvent être identifiées entre la fin du XIX ${ }^{e}$ siècle et le début des années 1990.

13 Entre 1890 et 1930, six sociétés sont créées à Frontignan (1890), Sète (1902, puis une seconde en 1921), Agde (1903), Palavas (1928), Béziers (1929). L'installation des sociétés signale à la fois un désir d'organisation de la pratique, un resserrement du lien social autour d'activités et de valeurs partagées et l'établissement d'une régulation sociale par les acteurs de la joute au sein de la joute et autour de la joute. Mais, la création d'une société ne se réalise qu'à partir d'une certaine densité de jouteurs et une trop grande densité entraîne une mobilité et la création d'une nouvelle société comme à Sète en 1921. Des relations se développent entre les sociétés pour l'organisation de tournois et des solidarités d'un niveau supérieur - celui des organisations - s'installent dans le champ du lien social. L'avènement des sociétés de joute marque le passage d'un lien social vivace et peu structurant à un lien social institué et structuré. La pérennité se substitue à la périodicité, le formel absorbe l'informel, l'organisation relaie l'individu. Cette transformation marque une étape dans l'institutionnalisation de nouveaux rapports entre le jouteur et la pratique : un intermédiaire s'est glissé, une mise à distance est introduite. L'organisation ne prendra que davantage de poids dans le mode d'organisation de la pratique et dans la gestion des relations sociales au sein de la pratique en s'installant comme l'incontournable interlocuteur reléguant le jouteur du rang d'acteur à celui d'agent.

Une deuxième période de création de sociétés s'amorce à l'issue de la seconde guerre mondiale. Deux sociétés sont créées à Mèze (1945) et à Sète (1946) dans la perspective de la création de la Fédération de joutes languedociennes qui intervient en 1946. Cette création marque une nouvelle étape dans le processus d'institutionnalisation de la 
joute. En imposant l'obligation de la licence pour les jouteurs, en hiérarchisant les jouteurs en quatre catégories de niveau et deux catégories de poids, en promouvant des règles pour le championnat de France, cet organe redéfinit les règles du jeu et, par là même, remodèle les relations développées par la pratique. Un degré supplémentaire est franchi, un transfert de charge est amorcé, une délégation de pouvoir et de représentation est mise en place. Le trajet du lien social traduit un déplacement du pouvoir de décision de l'individu à l'organisation et une régulation de la concurrence par la mise en place d'une gestion de la compétition et l'imposition d'une réglementation.

15 La troisième période où se concentrent six créations - Sète (1977, 1980, 1982), Frontignan (1989), Mèze (1981), Le Grau du Roi (1972) - démarre au début des années 80. Des modifications réglementaires tendent à limiter le nombre de jouteurs invités pour chaque société dans le cadre d'un tournoi local. Cette limitation a eu pour conséquence d'accroître la concurrence et de la déplacer au sein de chaque société en obligeant à des arbitrages internes pour la participation des membres aux tournois. La réponse à la limitation de cette densité est la constitution de nouveaux foyers de joute. Cette régulation morcelle le groupe des pratiquants en développant de nouveaux foyers de pratique où se recréent des relations. Ces créations sont le fait de jouteurs qui pour des raisons de différents ordres - affinitaires, concurrence, identité - inaugurent un univers de la joute. Ils attirent des jouteurs en provenance d'autres sociétés, installant la mobilité pour échapper à la densité.

Depuis le début du siècle, le jeu de forces entre mobilité et densité travaille la pratique de la joute en introduisant de nouveaux mécanismes de régulation du lien social. La concurrence mais également les formes compétitives renouvellent la nature de la communication en modifiant les modes et les espaces d'expression. Au cours de cette évolution, des mécanismes normatifs ont été introduits, ils ont pour nom: la réglementation, la ritualisation de la compétition, la logique de la hiérarchisation et de la classification. Non seulement ces mécanismes transforment l'organisation de la pratique mais ils instillent des normes et des repères distincts en rupture avec les représentations habituelles de la sociabilité dans ces sociétés. Les liens sociaux dans la pratique de la joute se recomposent à partir de comportements de moins en moins homogènes traduisant une transformation des mentalités et l'appropriation de valeurs sportives.

La « sportivisation » ou le paradigme de la hiérarchie et du classement

Dans une perspective sociologique structuraliste, la relation interindividuelle constitutive du lien social peut s'établir dans l'opposition. Caractérisée par le conflit, cette notion renvoie à une dimension première de la sociologie de Pierre Bourdieu dans la conquête de l'espace social et de sa distribution en classes, observées dans un rapport de sens et de domination symbolique pour la répartition des agents. Cette approche suppose qu'il existe un enjeu spécifique, générateur d'un rapport de force où se marque une identité et s'établit une dynamique. Chacun essaye de se positionner comme leader. L'espace social devient un ensemble de positions, il se parcellise en une multitude de sites différenciés où l'enjeu devient l'appropriation de "biens rares", symbole d'un statut social élevé. Dans ce contexte, deux éléments d'observation - la "sportivisation » et l'espace géographique des jouteurs - rendent compte de la construction et de la transformation du lien social dans la communauté des jouteurs. 

symbolique, en champion statutaire. La victoire erratique d'hier est balayée par l'ascèse d'aujourd'hui, conséquence d'aspects techniques et tactiques qui construisent le champion dans le temps et dans la durée tout en l'affectant de l'esprit de gagneur. Esprit qu'il forge dès le plus jeune âge comme culture systématique ${ }^{3}$ et non plus fortuite. Le système de valeurs évolue et passe d'une représentation commune du champion où il s'évertuait à vaincre par le beau geste et le courage, à un champion techniciste où s'effacent ces valeurs symboliques au profit de valeurs compétitives exclusives qui renforcent le pouvoir des jouteurs lourds. La compétition atténue l'esthétique et l'affectif. Dans cette approche classifiante et hiérarchisante qui structure l'espace social des jouteurs, le corps en tant que capital, devient l'instrument privilégié d'expression permettant d'établir les positions réciproques de chacun au sein des sociétés. Dans son engagement physique, le jouteur met en jeu ce capital acquis comme propriété significative pour lui dans la conquête de sa condition sociale. Le corps n'est plus seulement un construit physique représentatif d'un acteur de la joute, il devient un lieu d'échange d'où va dépendre un statut social. De fait, la catégorisation procure aux classes dominantes, les jouteurs lourds, une légitimité croissante. Ils vont s'octroyer d'autant plus facilement les enjeux de l'espace des joutes qu'ils détiennent des capitaux symboliques et culturels (par le corps notamment) d'honneur et de prestige. Ils s'imposent comme dominants légitimes, renforcés par l'instauration de ce système. La structure "supérieure ", reconnue comme l'élite des sociétés, les lourds, s'inscrit dans une configuration symbolique forte qui participe à renouveler le lien social, à le transformer dans la durée. Les relations, sous-jacentes du capital social dont chacun dispose, sont modifiées et s'établissent différemment. On se côtoie d'autant plus entre champions ou représentants directs d'une société, que l'on se retrouve systématiquement sur la tintaine chaque semaine.

20 D'autre part, une autre caractéristique de la «sportivisation » tend à accroître ce phénomène. Le nombre de jouteurs augmentant, la fédération a du limiter le nombre d'inscrit par société pour la participation aux tournois (fin des années 80). Désormais, seuls cinq jouteurs ont cet honneur. Ils assument la représentativité de l'ensemble des jouteurs, des valeurs de la société et tiennent lieu d'emblème. Ils sont les meilleurs. La forme symbolique de la représentation de la joute construit une hiérarchisation des valeurs. Celle-ci redessine un état nouveau dans la structuration du groupe des jouteurs au sein même de chaque société. Un rapport de force vertical s'établit entre eux et la participation aux tournois s'érige comme objectif premier. L'émergence de certains conflits est sous-jacente à ce nouvel ordre et s'inscrit comme la possibilité ou non d'un 
positionnement social sur l'échelle des représentations et des valeurs du groupe. Les relations en sont différenciées et redéfinissent l'ensemble du réseau interne d'échanges. Participer à la joute dans un champ de concurrence nouvellement défini par la règle revient à l'acquisition d'une reconnaissance commune, synonyme de rang sur l'échelle sociale des jouteurs. Cela participe de stratégies de positionnement qui se définissent dans "les relations objectives entretenues par les agents sociaux dans un champ " (Durand J.-P., Weil R., 1989: 195) et qui nécessitent la mobilisation des " pouvoirs fondamentaux qui sont les capitaux ». Le capital étant entendu comme la capacité pour tout agent social de déployer "toute énergie sociale susceptible de produire des effets et d'être utilisée (consciemment ou inconsciemment) comme instrument dans les concurrences sociales » (Ansart P., $1990: 101$ ). Cet enjeu primordial de participation devient la règle du jeu identifiable au fonctionnement de l'ensemble du champ. Laquelle participation se positionne au sommet des valeurs communes de la joute comme reconnaissance sociale. Si un champ fonctionne dans la mesure où il y a «des enjeux et des gens prêts à jouer le jeu dotés de l'habitus impliquant la connaissance et la reconnaissance des lois immanentes du jeu» (Bourdieu P., 1980 : 114), le champ de la joute existe comme tel. Mais il est structuré en fonction d'une concurrence qui, acceptée comme valeur classifiante et significative de positionnement par l'ensemble du groupe, devient déterminante pour l'appropriation du volume de capital et du statut social.

21 Les relations interindividuelles, génératrices du lien social qui existait avant l'instauration de nouvelles règles, ont évolué en fonction d'une lutte de classes. C'est l'ensemble du champ de la joute qui s'est restructuré par rapport à une hiérarchisation de valeurs qui a dépassée, sans l'annihiler, la « complicité objective qui réside dans la croyance en la valeur des enjeux de la lutte » (Durand J.-P., Weil R., 1989: 196) de la part des agents. Les rapports entre jouteurs qui s'établissaient sur une large communauté et qui fondaient un lien social étendu et rassembleur, se spécifient aujourd'hui. La domination des champions, reconnue par tous, séquentialise le lien social et le parcellise entre différents groupes de jouteurs, hiérarchisés par leur performance. La confrontation des forces en présence pour une participation plus forte à la pratique malgré une déontologie commune, répartit les agents dans l'espace social de façon arbitraire. Cet arbitraire légitimé par l'institutionnalisation assoie la domination d'une classe déjà prégnante.

Dans cet éclairage structuraliste, le lien social subit des transformations liées aux perturbations de la communauté des jouteurs et engendrées par le changement institutionnel. Il s'est reconstruit sur ces rapports de lutte traduits par l'appropriation et l'utilisation des capitaux disponibles dans le champ, pour l'obtention des enjeux circulants, sous la forme de participation systématique aux tournois. Le lien social se fragmente au sein du système "joute » et au sein de chaque société. Il permet de véhiculer le volume de capital pour l'élaboration de stratégies de distinction permettant le classement social dans la structure.

L'espace géographique des jouteurs : une opposition ethnique et socioprofessionnelle structurant le lien social

Un autre élément d'observation permet de rendre compte de la trajectoire du lien social constitué dans l'opposition: l'espace géographique des jouteurs. En effet, la situation géographique qui fonde la communauté des jouteurs est liée à deux aspects. Un premier aspect, ethnique, relève d'une répartition de classes basée sur des origines 
distinctes : le littoral languedocien s'étant construit sur un brassage ethnique culturel. Les immigrés italiens ont investi les côtes languedociennes dès le XIX ${ }^{e}$ siècle et se sont répartis sur tout le front de mer (Agde, Aigue Morte, Sète, Grau du roi). A Sète, haut lieu d'activité de la joute, la configuration spatiale de la ville s'organise en fonction d'un rapport de force originel. Français, italiens, marocains se sont rassemblés et fixés dans l'espace géographique de la cité. Le " quartier Haut ", le " quartier des Bordigues » ou celui de la «Pointe Courte » sont, en constituant des entités ethniques et définissant des quartiers, autant de lieux significatifs de conflits où « la structure d'interaction entre les hommes est marquée par le contexte spatial à travers lequel elle s'exprime » (Fischer G.N., 1985). Dans ce champ structurel, le lien social s'est construit à travers l'histoire par des confrontations ethniques et demeure aujourd'hui représentatif des différences culturelles du milieu de la joute.

Le deuxième aspect, également conflictuel, s'est établi dans un rapport de force où le pouvoir du capital économique, fonction de "l'intérêt économique qui a cours à un moment donné » (Durand J.-P., Weil R., 1989 : 194), a structuré l'espace géographique et social des jouteurs. Etre jouteur, c'est s'affirmer dans une dépendance à l'eau et une appartenance à un groupe défini: les pêcheurs. L'identification au groupe des jouteurs passe par la communion avec cette catégorie professionnelle qui a longtemps exercé une domination symbolique « ceux qui dominent le champ ayant les moyens de le faire fonctionner à leur profit » (Bourdieu P. 1980 : 136). Mais, l'avènement d'un secteur tertiaire dominant ${ }^{4} \mathrm{a}$ enseveli dans un contexte socioprofessionnel hétérogène le monde de la pêche. De surcroît, la diminution du capital économique des pêcheurs et le pouvoir qu'il véhiculait ont affaibli la position de ce groupe. Dans ce contexte de transformation socioprofessionnel, l'espace social s'est modifié selon de nouvelles lignes de force. La micro-économie sétoise s'est calquée sur la répartition socioprofessionnelle globale du pays, avec un secteur maritime évocateur d'une activité de pêche conséquente. Le lien social, s'il s'est transformé dans ces conflits de classes, rend toujours compte des relations socioprofessionnelles actuelles. Par exemple, il se réifie à travers l'apparat vestimentaire des jouteurs de la société des pêcheurs de Sète Môles qui, lors de leur tournoi annuel amical célébrant la Saint Pierre, patron des pêcheurs, montent fièrement sur la tintaine revêtus de leur maillot de marins. Les conflits ethniques et socioprofessionnels s'inscrivent dans la réalité sociohistorique des joutes et structurent, dans des rapports d'opposition, la répartition de l'espace social. La lutte des classes socioprofessionnelles devient significative de la construction du lien social. Dans cet espace, les relations sociales se développent à travers des lignes tangentielles qui tracent le réseau relationnel et la distribution géographique des agents selon un mode culturel et professionnel.

Ainsi, selon la perspective sociologique structuraliste, le lien social se construit et évolue à partir de situations d'opposition. Mais, si le système «joute» fonctionne aujourd'hui sur des rapports conflictuels qui orientent les relations interindividuelles et si certaines valeurs se fondent sur le domaine compétitif, technique et élitiste, il dépasse l'opposition grâce à un registre de valeurs communes qui ont traversé l'histoire et s'attachent à la pratique. Il s'agit d'observer le lien social, non plus comme émergent du conflit, mais comme perceptible dans la durée et constamment renouvelé dans le changement.

Entre sociologie dynamique et anthropologie sociale : le lien social dans le changement 
27 Par une approche sociologique qui s'oppose aux faits de structures et qui s'incarne dans une dynamique de changement, il est possible de porter un tout autre regard sur le lien social dans le système des joutes languedociennes. En effet, la conception sociologique dynamique propose une réflexion sur le fonctionnement de la société où la permanence de la structure s'efface devant son caractère transitionnel. C'est l'ensemble des processus de mouvement dans leur diversité qu'il faut observer afin de pouvoir rendre compte, par la multiplication d'aspects repérés, d'une dynamique dans l'évolution et non plus d'une reproduction unifiée des structures. Selon cette perspective, l'antériorité génératrice d'un état, structuré par ses antécédents, ne peut être, de fait, qu'un passage puisque transformée à nouveau par sa postériorité. Le changement ne doit plus être considéré comme «la destruction d'une identité qui serait caractérisée par un état d'équilibre et d'harmonie " (Laplantine F., 1987: 140) mais comme un contact entre deux états où l'un se nourrit de l'autre par et dans ce changement. Une lecture de cette "anthropo-logique " à travers la société des jouteurs permet de comprendre le changement, sa nature et son caractère coalescent avec le lien social.

D'un changement conjoncturel à un changement plus radical : quelle place pour le lien social?

L'histoire des joutes consacre trois siècles d'existence aux bords des canaux languedociens. Cette histoire est féconde de sens, de valeurs, de significations, de représentations qui ont traversé le temps dans et par le lien social. Ce dernier est le mécanisme catalyseur de tous les acteurs sociaux qui, au cours des siècles et des périodes de l'histoire, ont façonné, modelé et transformé la société des jouteurs. Il devient le témoin discret de connexions complexes et subtiles qui peuvent révéler le changement à un niveau local souvent caché. Il opère sur le latent, l'inexprimé ou l'informel, rendant ainsi son rôle significatif d'une réelle dynamique sociale entre les acteurs sociaux. Le lien social s'inscrit dans les moments fugitifs, les rencontres fortuites aussi bien que dans les manifestations commémoratives et structurées ${ }^{5}$. En fait, sous cet angle d'approche dynamique, il constitue un mouvement relationnel perpétuel, fécond de sens et d'interprétation cachés qui dévoilent les «instruments conceptuels " (Ansart P., 1990:55) des sociétés de joutes, autant dans leur connotation traditionnelle que moderne. Car, l'analyse du changement, décodé à travers le lien social, relève de la dichotomie tradition/modernité. Dépouillée par les courants sociologiques et anthropologiques, cette dualité s'est souvent appliquée à fondre l'objet dévitalisé dans un moule théorique qui, au delà de légitimer son existence, masquait le réel. Le propre de la vision anthropologique de G. Balandier est, au contraire, "d'appréhender la réalité derrière les apparences » (Gosselin G., 1993: 43) et de contextualiser l'objet d'étude.

29 Ainsi, du "plastron de bois qui leur recouvrait tout le corps » (Blanc L.P., 1968:6) au bonnet phrygien de la révolution, du marinier de pêche à la barque de résine, du costume d'apparat à la tenue blanche, le monde de la joute s'est transformé au gré des faits historiques passés et a perduré dans notre modernité cimenté par un lien social grégaire. C'est du moins, à l'échelle du temps, l'interprétation qui peut être faite du changement. Subi par les sociétés de joutes, celui-ci tient lieu de variations émanant des «turbulences » et de "l'effervescence » des crises qu'elles ont traversées. Mais, en aucun cas, le changement ne peut être apprécié comme radical puisque les transformations vécues, jusqu'à présent, n'étaient que conjoncturelles et n'avaient pas affecté «l'esprit» de la joute dans sa symbolique. C'est en cela que l'on peut 
caractériser le système « joute » de traditionnel puisque répondant de sa permanence à travers le temps par des mécanismes marqués du sceau de la tradition. Mémoire orale, conservation du patrimoine, «transmission par des procédures très codées du savoir le plus valorisé » (Balandier G., 1988 : 92), sont autant d'éléments qui participent à cette lecture traditionnelle et qui définissent l'identité culturelle des joutes languedociennes. Pourtant, l'avènement d'une structure "sportivisante " et d'une modélisation du champion en tant que compétiteur semble faire apparaitre depuis une cinquantaine d'années un étiolement rapide des valeurs traditionnelles qui structuraient le système " joute ». La tradition, portée par le lien social, se trouve confronter à cet élément qui s'inscrit subrepticement comme créateur d'une dynamique de rupture. Rupture déclencheuse de mécanismes dont on ne maîtrise pas l'importance et les conséquences. La docimologie s'est octroyée l'espace des pratiques et s'érige comme initiatrice d'une " société de plus en plus métrisée » (Balandier G., 1991 : 117). Les relations de fêtes, les relations de tournois, les relations d'entraînements, constitutives du lien social, sont modifiées et remodelées selon un nouvel ordre de valeurs. Ces valeurs incarnent une modernité de l'individu construisant une société parcellisée, sectorisée où le lien social apparaît dans le superficiel et la labilité qui tendent à annihiler « l'esprit traditionnel ». Certains indicateurs dévoilent cette tendance comme, par exemple, le désintérêt des jouteurs à participer aux rites festifs d'après tournois. Combien sont-ils désormais à "ramener » en défilant le glorieux vainqueur au siège de la société ? Combien sontelles ces voix languedociennes bigarrées des jouteurs chantant au détour d'un bar pour fêter le champion? De moins en moins.

31 Si le monde de la joute se fond dans le carcan solipsiste de la société dite post-moderne qui s'édifie dans le «mouvant, l'incertitude et le complexe » (ibid., 121), il risque d'être phagocyté par cette modernité au point d'en perdre ses repères. Si l'on considère que la nature du changement, actuellement subi par le système "joute ", revêt un caractère plus radical qui s'oppose aux transformations générées à l'échelle du temps et liées aux moments conjoncturels des époques, alors le système traditionnel des valeurs, des représentations et la symbolique commune qui en découle pourrait se transformer rapidement au risque d'un effritement entrainant la dissolution du lien social. Cette situation, par son caractère coalescent au changement, risquerait de réduire la tradition à « ce qui s'avérerait être de plus en plus de la fiction » (Camy J., 1985: 75).

Mais, il convient d'être prudent car cette orientation "moderniste» des joutes languedociennes correspond plus à une logique de «brouillage» effectuée par la modernité qu'à une disparition proche de cette pratique. Celle-ci, érigée en société traditionnelle, possède les clés de la continuité. Forgées dans l'acceptation constante d'un désordre « reconnu comme inhérent au mouvement de toute vie » (Balandier G., 1988 : 123), elles permettent d'entrouvrir la porte au changement sans pour autant souffrir d'un effacement total de l'existant. Là s'impose la force et la puissance de la tradition. Les hommes, les jouteurs, par leur croyance commune dans l'immortalité de la joute, dans sa signification symbolique, sa grandeur et sa magnificence, constituent la chair d'une mémoire collective qu'on ne peut corroder même dans une radicalisation extrême. Cette mémoire vit par le témoignage des ascendants aux descendants et s'incarne dans le lien social, constitutif de cette continuité. Inséré comme pilier du système "joute ", le témoignage intervient comme un ajustement permanent au changement. Confrontés à ce dernier, les jouteurs « vont utiliser l'ancienne structure, la maintenir tout en la modifiant à leur profit » (Balandier G., $1971: 225)$. 
33 Ainsi traité sous cet angle anthropologique, le lien social apparaît comme le médiateur qui permet au système "joute» de répondre aux soubresauts insufflés par la modernité. Celle-ci, représentée dans la joute par l'aspect compétitif drainant des valeurs individuelles, chahute l'existant de façon plus radicale que les vagues de changement précédemment rencontrées. Dans cette «impression que la postmodernité est une rupture complète, une sorte de nouveau commencement absolu » (Balandier G., 1991 : 121), il faut se garder de considérer un quelconque caractère final où la tradition perdrait de sa constitution. Il s'agit pour les jouteurs de repérer et de finaliser des nouvelles formes de lien social qui, de façon continuelle, agiront comme les garantes d'une société plurielle alliant devant l'éternel tradition et modernité.

Le lien social : un objet sociologique aux multiples facettes

Etudier le lien social consiste à embrasser tout l'objet de la sociologie. Aussi, au risque de se perdre dans des considérations trop générales, le parti pris de resserrer l'observation et de choisir une stratégie a été osé. Il postule que la dynamique du lien social est plus heuristique que son état (Xiberras M., 1995). Dans cette perspective, un choix méthodologique s'imposait pour couvrir la trajectoire des relations sociales au sein d'une pratique de jeux traditionnels. Il visait à combiner différentes approches sociologiques qui modélisent le lien social à partir d'un cadre théorique articulant mécanismes et concepts. Quatre approches ont permis d'aborder et de restituer des aspects de la dynamique du lien social dans le monde de la joute. Eclairage plus que révélation, ce "vagabondage» montre combien la capture du lien social est une entreprise difficile si l'on veut éviter la tentation totalisante de la fixité, de la régulation et du contrôle. Au terme de cette tentative, l'image d'une métamorphose évoque la recomposition du lien social à partir de règles qui échappent à l'individu pour se concentrer sur l'institution. Dans la pratique de la joute, le lien social a connu différentes phases. La plus ancienne pourrait se définir comme une phase d'émancipation du lien social, elle s'achève à l'aube $d u x^{e}$ siècle. Succède à cette première phase, une période de cristallisation du lien social dans les pratiques de joute, elle correspond à l'essor des sociétés. Avec la «sportivisation" croissante de cette pratique, les polarités constitutives des relations sociales fluctuent puis s'inversent : les relations de proximité sont phagocytées par les relations nouées sur et autour de l'objet technique. La compétition et son arsenal normatif ont définitivement remplacé le défi et l'initiative individuelle. L'adhésion aux valeurs et au patrimoine de la joute se réalise selon un mode d'appropriation de plus en plus personnel façonnant une vision singulière et une approche sélective de la pratique. Insensiblement, l'héritage symbolique et traditionnel est gommé au profit d'une orientation plus technicienne entraînant des transformations dans le contenu et la nature du lien social. Cette évolution pose avec acuité la question de la signification de la pratique écartelée entre la gestion d'un héritage et celle d'un devenir. Partir du sens pour reconstruire ou fortifier le lien social de la joute pourrait s'avérer être une stratégie gagnante afin d'infléchir la dynamique des relations dans la pratique de la joute. 


\section{BIBLIOGRAPHIE}

Agulhon M. (1977). Le cercle de la France bourgeoise. 1810-1848, Paris, Armand Colin.

Ansart P. (1990). Les sociologies contemporaines. Paris, Seuil.

Arnaud P. (1987). (sous la direction), Les athlètes de la République. Gymnastique, sport et idéologie républicaine. 1870/1914, Toulouse, Privat.

Balandier G. (1971). Sens et puissance, les dynamiques sociales. Paris, PUF.

Balandier G. (1988). Le désordre. Paris, Fayard.

Balandier G. (1991). Regard anthropologique sur la modernité et post-modernité, in Corps, religion, société, CREA, Lyon, PUL.

Blanc L.P. (1968). Les joutes à Sète, Sète, Centre Culturel Sétois.

Bolle de Bal M. (Ed.) (1996). Voyage au coeur des Sciences Humaines. De la reliance. Tome 2 : Reliance et Pratiques, Paris, L'Harmattan.

Bourdieu P. (1980). Questions de sociologie. Paris, Editions de Minuit.

Callede J.P. (1985). La sociabilité sportive et l'expression identitaire, in Ethnologie Française, XV, 4, p.327-344.

Callede J.P. (1987). L'esprit sportif, Bordeaux, PUB.

Camy J. (1985). (sous la direction), Identités Givordines, Rapport à la mission du patrimoine ethnologique, Ministère de la Culture.

Durand J.-P. et Weil R. (1989). Sociologie contemporaine. Paris, Vigot.

Durkheim E. (1976). Le suicide, Paris, P.U.F.

Durkheim E. (1977). Les règles de la méthode sociologique, Paris, P.U.F.

Durkheim E. (1978). De la division du travail social, Paris, P.U.F.

Durkheim E. (1979). Les formes élémentaires de la vie religieuse, Paris, P.U.F.

Fischer G.N. (1981). La psychosociologie de l'espace. Paris, PUF.

Gosselin G. (1993). Les nouveaux enjeux de l'anthropologie, autour de Georges Balandier. Paris, L'Harmattan.

Grafmeyer Y., Joseph I. (1979). L'Ecole de Chicago, Paris, Champ Urbain.

Hubscher R. et Durry J. et Jeu B. (1992). L'histoire en mouvements. Le sport dans la société française (XIX ème $\left.-\mathrm{XX}^{\text {ème }}\right)$, Paris, Armand Colin

Laplantine F. (1987). L'anthropologie. Paris, Seghers.

Pigeassou C. (1996). Le développement des joutes languedociennes : de la tradition à la modernité, in Sport, éducation et art. $\mathrm{XIX}^{\mathrm{e}}-\mathrm{XX}^{\mathrm{e}}$ siècles, Arnaud P., Terret T. (sous la direction), Paris, Editions CTHS, p. 373-384.

Pouget V. (1993). Nos joutes nautiques. Chronique de la société nautique des jouteurs agathois, Pèzenas, Editions Domens. 
Simmel G. (1979). Digressions sur l'étranger, in L'Ecole de Chicago, Grafmeyer Y., Joseph I., Paris, Champ Urbain.

Tönnies F. (1963). Community and Society, New York, Harper Torchbook.

Weber M. (1964). L'éthique protestante et l'esprit du capitalisme, Paris, Plon.

Xiberras M. (1993). Les théories de l'exclusion, Paris, Méridens Klincksieck.

\section{NOTES}

1. Cette cérémonie a pour objectif de désigner parmi les troupes des mariés, celle qui aura l'honneur de jouter contre la jeunesse. Elle consiste à mettre en concurrence plusieurs troupes de mariés, selon des procédures établies, pour se saisir d'un pavois préalablement disposé au bout d'une lance.

2. Dès 1946 deux catégories distinctes sont créées par la ligue languedocienne. Les moins de $80 \mathrm{~kg}$ sont dénommés « légers » et les plus de $80 \mathrm{~kg}$ sont dénommés « lourds ». 3. En 1977 est créée la première école de joutes. Elle s’institue très vite comme structure de modélisation du champion notamment en créant dès 1981 le championnat de France pour enfants. Ils joutent dès l'âge de 4 ans.

4. La ville de Sète a compté jusqu'à deux mille dockers sur les quais du port dans les années 50.

5. Toute rencontre entre jouteurs, qu'elle soit fortuite ou lors d'un tournoi, procède de relation amicale autour d'un seul sujet de discussion: la joute. Cela participe à un renforcement du lien social qui prend tout son sens dans ces moments là.

\section{RÉSUMÉS}

Le lien social est un objet central dans la sociologie. Son étude se heurte à son dynamisme et aux différents registres de ces mécanismes. L'exploration de ces deux aspects a été menée avec le support de plusieurs cadres de référence sociologiques dans le contexte d'une pratique de jeu traditionnel : la joute.

The social bond is a main topic in the field of the sociology. The study of such a theme meets with the dynamism and the diversity of the processes involved. The paper explores these aspects with the support of various sociological theories in the context of water tournament.

\section{INDEX}

Keywords : sport, relationship, social bond, sociology, water tournament Mots-clés : lien social, relations sociales, joutes, sociologie 
AUTEURS

\section{CHARLES PIGEASSOU}

Faculté des Sciences du Sport et de l'Education Physique. Université de Montpellier, Equipe « Corps et Culture»

JÉRÔME PRUNEAU

Faculté des Sciences du Sport et de l'Education Physique. Université de Montpellier, Equipe « Corps et Culture » 